\title{
On the Dirichlet Problem for the Stokes System in Multiply Connected Domains
}

\author{
Alberto Cialdea, Vita Leonessa, and Angelica Malaspina \\ Department of Mathematics, Computer Science and Economics, University of Basilicata, Viale dell'Ateneo Lucano 10, \\ 85100 Potenza, Italy
}

Correspondence should be addressed to Alberto Cialdea; cialdea@email.it

Received 24 April 2012; Accepted 28 November 2012

Academic Editor: Chun-Lei Tang

Copyright (C) 2013 Alberto Cialdea et al. This is an open access article distributed under the Creative Commons Attribution License, which permits unrestricted use, distribution, and reproduction in any medium, provided the original work is properly cited.

The Dirichlet problem for the Stokes system in a multiply connected domain of $\mathbb{R}^{n}(n \geq 2)$ is considered in the present paper. We give the necessary and sufficient conditions for the representability of the solution by means of a simple layer hydrodynamic potential, instead of the classical double layer hydrodynamic potential.

\section{Introduction}

Potential theory methods have been employed for a long time in the study of boundary value problems. In particular they were widely used in BVPs for the Stokes system, starting from $[1,2]$.

Recently some papers have used the integral representations of solutions for studying some BVPs for the Stokes system also in multiply connected domains [3-8]. All these papers concern the double layer hydrodynamic potential approach for the Dirichlet problem and the simple layer hydrodynamic potential approach for the traction problem.

The aim of the present paper is to investigate a different integral representation for the Dirichlet problem for the Stokes system in a multiply connected bounded domain of $\mathbb{R}^{n}(n \geq 2)$. Namely, we consider the simple layer potential approach for the Dirichlet problem in a domain

$$
\Omega=\Omega_{0} \backslash \bigcup_{j=1}^{m} \bar{\Omega}_{j},
$$

where $\Omega_{j}(j=0, \ldots, m)$ are suitable domains with connected boundaries in $C^{1, \lambda}, \lambda \in(0,1]$.

We use a new method which hinges on a singular integral system in which the unknown is a usual vector valued function, while the data is a vector whose components are differential forms.
The paper is organized as follows. In Section 2 we give an outlook of the method with a brief description of some previous results.

After the preliminary Section 3, in Section 4 we study in detail the case $n=2$, where some particular phenomena appear.

Section 5 is devoted to determine the eigenspace of a certain singular integral system in which the unknowns are differential forms of degree $n-2$ on $\partial \Omega$. In the same section, we recall some known results concerning the eigenspaces of some classical integral systems.

In Section 6 we construct a left reduction for the singular integral system under study. Such a singular integral system is equivalent in a precise sense to the Fredholm system obtained through the reduction.

Finally, in the last section, we find the solution of the Dirichlet problem for the Stokes system in a multiply connected domain by means of a simple layer hydrodynamic potential.

The main result is that, given $f \in\left[W^{1, p}(\partial \Omega)\right]^{n}$, we can represent the solution of the Dirichlet problem

$$
\begin{aligned}
\mu \Delta v=\nabla r & \text { in } \Omega, \\
\operatorname{div} v=0 & \text { in } \Omega, \\
v=f & \text { on } \partial \Omega,
\end{aligned}
$$


by means of a simple layer hydrodynamic potential if, and only if, the conditions

$$
\int_{\partial \Omega_{j}} f \cdot v d \sigma=0, \quad j=0,1, \ldots, m
$$

are satisfied ( $v$ being the outwards unit normal on $\partial \Omega$ ). Moreover, if the data $f$ satisfies only the condition

$$
\int_{\partial \Omega} f \cdot v d \sigma=0
$$

(which is necessary for the existence of a solution of the Dirichlet problem (2)) we show how to modify the integral representation of the solution (see Theorem 23).

\section{Sketch of the Method}

The aim of this section is to give a better understanding of the method we are going to use in the present paper.

We will do that by considering the Dirichlet problem for Laplace equation in a bounded simply connected domain $\Omega \subset$ $\mathbb{R}^{n}$, whose boundary we denote by $\Sigma$ as follows:

$$
\begin{array}{cc}
\Delta u=0 & \text { in } \Omega, \\
u=g & \text { on } \Sigma .
\end{array}
$$

Suppose that $g \in W^{1, p}(\Sigma), 1<p<\infty$. If we want to find the solution in the form of a simple layer potential whose density belongs to $L^{p}(\Sigma)$, we have to solve an integral equation of the first kind on $\Sigma$ as follows:

$$
\int_{\Sigma} \varphi(y) s(x, y) d \sigma_{y}=g(x), \quad x \in \Sigma,
$$

where $s(x, y)$ is the fundamental solution of Laplace equation

$$
s(x, y)= \begin{cases}-\frac{1}{2 \pi} \log \frac{1}{|x-y|}, & \text { if } n=2, \\ -\frac{1}{\omega_{n}(n-2)} \frac{1}{|x-y|^{n-2}}, & \text { if } n \geq 3 .\end{cases}
$$

In [9] a new method for discussing such an equation was proposed. Namely, the first step is to consider the differential (in the sense of the theory of differential forms) of both sides in (6). In this way we obtain the equation

$$
\int_{\Sigma} \varphi(y) d_{x}[s(x, y)] d \sigma_{y}=d g(x), \quad x \in \Sigma,
$$

in which we look for a solution $\varphi \in L^{p}(\Sigma)$.

The integral on the left hand side is a singular integral and it can be considered as a linear and continuous operator from $L^{p}(\Sigma)$ to $L_{1}^{p}(\Sigma)$ (we denote by $L_{h}^{p}(\Sigma)$ the space of the differential forms of degree $h$ whose coefficients belong to $L^{p}(\Sigma)$ in every local coordinate system).

It must be remarked that, if $n \geq 3$, the space in which we look for the solution of (8) and the space in which the data is given are different.

We recall that, if $B$ and $B^{\prime}$ are two Banach spaces and $S: B \rightarrow B^{\prime}$ is a continuous linear operator, $S$ can be reduced on the left if there exists a continuous linear operator $S^{\prime}$ : $B^{\prime} \rightarrow B$ such that $S^{\prime} S=I+T$, where $I$ stands for the identity operator on $B$, and $T: B \rightarrow B$ is compact. Analogously, one can define an operator $S$ reducible on the right. One of the main properties of such operators is that the equation $S \alpha=\beta$ has a solution if, and only if, $\langle\gamma, \beta\rangle=0$ for any $\gamma$ such that $S^{*} \gamma=0, S^{*}$ being the adjoint of $S$ (for more details see, e.g., $[10,11])$.

Let us denote by $S \varphi$ the left hand side of (8). In [9] a reducing operator $S^{\prime}$ was explicitly constructed. This implies that there exists a solution of (8) if, and only if, the compatibility conditions

$$
\int_{\Sigma} d g \wedge h=0
$$

are satisfied for any $h \in L_{n-2}^{q}(\Sigma)(q=p /(p-1))$ such that $S^{*} h=0$. Moreover one can show that $S^{*} h=0$ if, and only if, $h$ is a weakly closed form. Therefore the compatibility conditions (9) are satisfied, and there exists a solution $\varphi \in$ $L^{p}(\Sigma)$ of $(8)$.

A left reduction is said to be equivalent if $\mathrm{N}\left(S^{\prime}\right)=\{0\}$, where $\mathrm{N}\left(S^{\prime}\right)$ denotes the kernel of $S^{\prime}$ (see, e.g., [11, page 19-20]). Obviously this means that $S x=y$ if, and only if, $S^{\prime} S x=S^{\prime} y$. In [12] it was remarked that if $\mathrm{N}\left(S^{\prime} S\right)=\mathrm{N}(S)$, we still have a kind of equivalence. Indeed the coincidence of these two kernels implies the following fact: if $y$ is such that the equation $S x=y$ is solvable, then this equation is satisfied if, and only if, $S^{\prime} S x=S^{\prime} y$.

Since $\mathrm{N}\left(S^{\prime} S\right)=\mathrm{N}(S)$, then we have (8) equivalent to the Fredholm equation $S^{\prime} S \varphi=S^{\prime}(d g)$. These results lead to a simple layer potential theory for the Dirichlet problem (5).

As a consequence one can obtain also a double layer representation for the Neumann problem for Laplace equation [12].

A characteristic of this method is that it uses neither the theory of pseudodifferential operators nor the concept of hypersingular integrals.

This method has been used also for studying other BVPs. In particular in [13] it was used to study the Dirichlet and the Neumann problems in multiply connected domains. Among other things, an interesting by-product of these results was obtained as follows (see [13, Theorem 6.1]).

Let $u$ be a harmonic function of class $C^{1}(\bar{\Omega})$, where $\Omega$ is the multiple connected domain (1). There exists a 2-form $v$ conjugate to $u$ in $\Omega$ if, and only if,

$$
\int_{\partial \Omega_{j}} \frac{\partial u}{\partial v} d \sigma=0, \quad j=0,1, \ldots, m .
$$

An explicit integral expression for $v$ was also given. We recall that the 2 -form $v$ is conjugate to $u$ if $d u=\delta v, d v=0$.

The method has been applied to different BVPs for several PDEs (see [12-19]).

\section{Preliminaries}

In this paper $\Omega$ denotes an $(m+1)$-connected domain of $\mathbb{R}^{n}$ $(n \geq 2)$, that is an open-connected set of the form (1), where each $\Omega_{j}(j=0, \ldots, m)$ is a bounded domain of $\mathbb{R}^{n}$ with 
connected boundaries $\Sigma_{j} \in C^{1, \lambda}(\lambda \in(0,1])$, and such that $\bar{\Omega}_{j} \subset \Omega_{0}$ and $\bar{\Omega}_{j} \cap \bar{\Omega}_{k}=\emptyset, j, k=1, \ldots, m, j \neq k$. Let $v$ be the outwards unit normal on the boundary $\Sigma=\partial \Omega$.

We consider the classical Stokes system for the incompressible viscous fluid

$$
\begin{gathered}
\mu \Delta u=\nabla p, \quad \text { in } \Omega, \\
\operatorname{div} u=0,
\end{gathered}
$$

where the unknowns $u=\left(u_{1}, \ldots, u_{n}\right)$ and $p=p(x)$ are the velocity and pressure of the fluid flow, respectively, and the constant $\mu>0$ is the kinematic viscosity of the fluid. A fundamental solution for this system is given by the pair of fundamental velocity tensor and its associated pressure vector

$$
\begin{aligned}
& \gamma_{i j}(x, y)=\left\{\begin{aligned}
-\frac{1}{4 \pi \mu}\left[\delta_{i j} \log \frac{1}{|x-y|}\right. & \\
\left.+\frac{\left(x_{i}-y_{i}\right)\left(x_{j}-y_{j}\right)}{|x-y|^{2}}\right], & \text { if } n=2, \\
-\frac{1}{2 \omega_{n} \mu}\left[\frac{\delta_{i j}}{n-2} \frac{1}{|x-y|^{n-2}}\right. & \\
\left.+\frac{\left(x_{i}-y_{i}\right)\left(x_{j}-y_{j}\right)}{|x-y|^{n}}\right], & \text { if } n \geq 3,
\end{aligned}\right. \\
& \varepsilon_{j}(x, y)=-\frac{1}{\omega_{n}} \frac{x_{j}-y_{j}}{|x-y|^{n}},
\end{aligned}
$$

$(i, j=1, \ldots, n), \omega_{n}$ being the hypersurface measure of the unit sphere in $\mathbb{R}^{n}$. For a solution $(u, p)$ of $(11)$ we consider the following classical boundary operators:

$$
\begin{gathered}
T_{j} u=\left[-\delta_{i j} p+\mu\left(\partial_{j} u_{i}+\partial_{i} u_{j}\right)\right] v_{i}, \\
T_{j}^{\prime} u=\left[\delta_{i j} p+\mu\left(\partial_{j} u_{i}+\partial_{i} u_{j}\right)\right] v_{i},
\end{gathered}
$$

Through this paper, $p$ indicates a real number such that $1<p<+\infty$. We denote by $\left[L^{p}(\Sigma)\right]^{n}$ the space of all measurable vector-valued functions $u=\left(u_{1}, \ldots, u_{n}\right)$ such that $\left|u_{j}\right|^{p}$ is integrable over $\Sigma(j=1, \ldots, n)$. If $h$ is any nonnegative integer, $L_{h}^{p}(\Sigma)$ is the vector space of all differential forms of degree $h$ (briefly $h$-forms) defined on $\Sigma$ such that their components are integrable functions belonging to $L^{p}(\Sigma)$ in a coordinate system of class $C^{1}$ and consequently in every coordinate system of class $C^{1}$. The space $\left[L_{h}^{p}(\Sigma)\right]^{n}$ is constituted by the vectors $\left(v_{1}, \ldots, v_{n}\right)$ such that $v_{j}$ is a differential form of $L_{h}^{p}(\Sigma)(j=1, \ldots, n)$. $\left[W^{1, p}(\Sigma)\right]^{n}$ is the vector space of all measurable vector-valued functions $u=\left(u_{1}, \ldots, u_{n}\right)$ such that $u_{j}$ belongs to the Sobolev space $W^{1, p}(\Sigma)(j=1, \ldots, n)$.

The pair $(v, r)$ with components

$$
\begin{gathered}
v_{i}(x)=-\int_{\Sigma} \gamma_{i j}(x, y) \varphi_{j}(y) d \sigma_{y}, \quad i=1, \ldots, n, x \in \mathbb{R}^{n}, \\
r(x)=-\int_{\Sigma} \varepsilon_{j}(x, y) \varphi_{j}(y) d \sigma_{y}, \quad x \in \mathbb{R}^{n}
\end{gathered}
$$

is the simple layer hydrodynamic potential with density $\varphi$.

The pair $(w, q)$ with components

$$
w_{i}(x)=\int_{\Sigma} T_{j, y}^{\prime}\left[\gamma^{i}(x, y)\right] \psi_{j}(y) d \sigma_{y}, \quad i=1, \ldots, n, x \in \mathbb{R}^{n},
$$

$$
q(x)=2 \mu \int_{\Sigma} \frac{\partial}{\partial v_{y}}\left[\varepsilon_{j}(x, y)\right] \psi_{j}(y) d \sigma_{y}, \quad x \in \mathbb{R}^{n}
$$

is the double layer hydrodynamic potential with density $\psi$.

\section{On the Bidimensional Case}

It is wellknown that there are some exceptional plane domains in which no every harmonic function can be represented by a simple layer potential. The simplest example of this kind is given by the unit disk, for which one has

$$
\int_{|y|=1} \log |x-y| d s_{y}=0, \quad|x|<1 .
$$

It is also known that such domains do not occur in higher dimensions. For similar questions for the Laplace equation and the elasticity system, see [13, Section 3$]$ and [16, Section 4], respectively.

In this section we show that also for the Stokes system there are similar domains. We say that the boundary of the domain $\Omega$ is exceptional if there exists some constant vector which cannot be represented in $\Omega$ by a simple layer potential.

Denoting by $\Sigma_{R}$ the circle of radius $R$ centered at the origin, we have the following lemma.

Lemma 1. The circle $\Sigma_{R}$ with $R=\exp (1 / 2)$ is exceptional for the Stokes system.

Proof. Keeping in mind that (see, e.g., $[16$, Section 4])

$$
\begin{gathered}
\int_{\Sigma_{R}} \log |x-y| d s_{y}=2 \pi R \log R, \\
\int_{\Sigma_{R}} \frac{\left(x_{i}-y_{i}\right)\left(x_{j}-y_{j}\right)}{|x-y|^{2}} d s_{y}=\delta_{i j} \pi R, \quad|x|<R,
\end{gathered}
$$

we find

$$
\int_{\Sigma_{R}} \gamma_{i j}(x, y) d s_{y}=\frac{R}{4 \mu} \delta_{i j}(2 \log R-1), \quad|x|<R .
$$

Taking $R=\exp (1 / 2)$ we obtain the result.

Let us consider now the exceptional boundaries of not simply connected domains.

Proposition 2. Let $\Omega \subset \mathbb{R}^{2}$ be an $(m+1)$-connected domain. Denote by $\mathscr{P}$ the eigenspace in $\left[L^{p}(\Sigma)\right]^{2}$ of the singular integral system

$$
\int_{\Sigma} \varphi_{j}(y) \frac{\partial}{\partial s_{x}} \gamma_{i j}(x, y) d s_{y}=0, \quad \text { a.e. } x \in \Sigma, i=1,2 .
$$

Then $\operatorname{dim} \mathscr{P}=2(m+1)$. 
Proof. As in the proof of [16, Lemma 12], one can show that

$$
\frac{\partial}{\partial s_{x}} \gamma_{i j}(x, y)=\frac{1}{4 \pi \mu} \delta_{i j} \frac{\partial}{\partial s_{x}} \log |x-y|+\mathcal{O}\left(|y-x|^{h-1}\right),
$$

deduce that system (22) can be regularized to a Fredholm one, and see that its index is zero. Since the vectors $e_{i} \chi_{\Sigma_{j}}$ (by $\chi_{X}$ we denote the characteristic function of the set $X)(i=1,2, j=$ $0,1, \ldots, m)$ are the only eigensolutions of the adjoint system

$$
\int_{\Sigma} \varphi_{j}(y) \frac{\partial}{\partial s_{y}} \gamma_{i j}(x, y) d s_{y}=0, \quad \text { a.e. } x \in \Sigma, i=1,2,
$$

we have $\operatorname{dim} \mathscr{P}=2(m+1)$.

Theorem 3. Let $\Omega \subset \mathbb{R}^{2}$ be an $(m+1)$-connected domain. The following conditions are equivalent

(1) There exists a Hölder continuous vector function $\varphi \not \equiv 0$ such that

$$
\int_{\Sigma} \gamma(x, y) \varphi(y) d s_{y}=0, \quad x \in \Sigma
$$

(2) There exists a constant vector which cannot be represented in $\Omega$ by a simple layer potential;

(3) $\Sigma_{0}$ is exceptional.

(4) Let $\varphi_{1}, \ldots, \varphi_{2 m+2}$ be linearly independent vectors of $\mathscr{P}$ (see Proposition 2), and let $c_{j k}=\left(\alpha_{j k}, \beta_{j k}\right) \in \mathbb{R}^{2}$ be given by

$$
\int_{\Sigma} \gamma(x, y) \varphi_{j}(y) d s_{y}=c_{j k}
$$

$x \in \Sigma_{k}, j=1, \ldots, 2 m+2, k=0,1, \ldots, m$.

Then $\operatorname{det} \mathscr{C}=0$, where

$$
\mathscr{C}=\left(\begin{array}{ccc}
\alpha_{1,0} & \cdots & \alpha_{2 m+2,0} \\
\cdots & \cdots & \cdots \\
\alpha_{1, m} & \cdots & \alpha_{2 m+2, m} \\
\beta_{1,0} & \cdots & \beta_{2 m+2,0} \\
\cdots & \cdots & \cdots \\
\beta_{1, m} & \cdots & \beta_{2 m+2, m}
\end{array}\right) .
$$

Proof. The proof runs as in [16, Theorem 1] with obvious modifications. We omit the details.

\section{Some Eigenspaces}

We determine the structure of the kernel of a particular singular integral system. Namely, let us denote by $\mathcal{N}_{p}$ the space of $\psi \in\left[L_{n-2}^{p}(\Sigma)\right]^{n}$ such that

$$
\int_{\Sigma} \psi_{j}(y) \wedge d_{y}\left[\gamma_{i j}(x, y)\right]=0, \quad \text { a.e. on } \Sigma, i=1, \ldots, n \text {. }
$$

We begin by proving the following result.
Lemma 4. Let $u \in\left[C_{0}^{\infty}\left(\mathbb{R}^{n}\right)\right]^{n}$. Then, for any $x \in \mathbb{R}^{n}$,

$$
u_{i}(x)=\mu \int_{\mathbb{R}^{n}} \Delta u_{j}(y) \gamma_{i j}(x, y) d y+\int_{\mathbb{R}^{n}} \frac{\partial^{2} u_{j}(y)}{\partial y_{i} \partial y_{j}} s(x, y) d y,
$$

where $\gamma(x, y)$ and $s(x, y)$ are given by (12) and (7), respectively. Proof. By the well-known Stokes identity we have

$$
u_{i}(x)=\int_{\mathbb{R}^{n}} \Delta u_{i}(y) s(x, y) d y=\int_{\mathbb{R}^{n}} \Delta u_{j}(y) \delta_{i j} s(x, y) d y .
$$

Since, for every $n \neq 2,4$,

$$
\begin{gathered}
\frac{\left(x_{i}-y_{i}\right)\left(x_{j}-y_{j}\right)}{|x-y|^{n}}=\frac{1}{(4-n)(2-n)} \frac{\partial^{2}}{\partial y_{i} \partial y_{j}}|x-y|^{4-n} \\
-\delta_{i j} \omega_{n} s(x, y), \\
\gamma_{i j}(x, y)=\frac{\delta_{i j}}{2 \mu} s(x, y)-\frac{1}{2 \mu \omega_{n}} \frac{\left(x_{i}-y_{i}\right)\left(x_{j}-y_{j}\right)}{|x-y|^{n}}, \quad \forall n \geq 2,
\end{gathered}
$$

we can rewrite

$$
\begin{aligned}
\gamma_{i j}(x, y)= & \frac{\delta_{i j}}{\mu} s(x, y)-\frac{1}{2 \mu \omega_{n}} \frac{1}{(4-n)(2-n)} \frac{\partial^{2}}{\partial y_{i} \partial y_{j}} \\
& \times|x-y|^{4-n} .
\end{aligned}
$$

Then

$$
\begin{aligned}
& \delta_{i j} s(x, y)=\mu \gamma_{i j}(x, y)+\frac{1}{2 \omega_{n}(4-n)(2-n)} \frac{\partial^{2}}{\partial y_{i} \partial y_{j}}|x-y|^{4-n}, \\
& u_{i}(x)=\mu \int_{\mathbb{R}^{n}} \Delta u_{j}(y) \gamma_{i j}(x, y) d y
\end{aligned}
$$

$$
+\frac{1}{2 \omega_{n}(4-n)(2-n)} \int_{\mathbb{R}^{n}} \Delta u_{i}(y) \frac{\partial^{2}}{\partial y_{i} \partial y_{j}}|x-y|^{4-n} d y .
$$

Integrating by parts, it follows that the last integral is equal to

$$
\begin{gathered}
\frac{1}{2 \omega_{n}(4-n)(2-n)} \int_{\mathbb{R}^{n}} \frac{\partial^{2} u_{j}(y)}{\partial y_{i} \partial y_{j}} \Delta_{y}|x-y|^{4-n} d y \\
=\int_{\mathbb{R}^{n}} \frac{\partial^{2} u_{j}(y)}{\partial y_{i} \partial y_{j}} s(x, y) d y,
\end{gathered}
$$

since $\Delta_{y}|x-y|^{4-n}=2(4-n)|x-y|^{2-n}$. Then the claim holds for $n \neq 2,4$.

In the same manner it is possible to show formula (29) for $n=2$ and $n=4$ after observing that, if $n=2$, we have

$$
\begin{aligned}
\frac{\left(x_{i}-y_{i}\right)\left(x_{j}-y_{j}\right)}{|x-y|^{2}}= & \frac{1}{2} \frac{\partial^{2}}{\partial y_{i} \partial y_{j}}|x-y|^{2} \log |x-y| \\
& -\delta_{i j} \log |x-y|-\frac{1}{2} \delta_{i j},
\end{aligned}
$$


$\Delta_{y}|x-y|^{2} \log |x-y|=4(\log |x-y|+1)$, while, for $n=4$,

$$
\frac{\left(x_{i}-y_{i}\right)\left(x_{j}-y_{j}\right)}{|x-y|^{4}}=\frac{\delta_{i j}}{2|x-y|^{2}}+\frac{1}{2} \frac{\partial^{2}}{\partial y_{i} \partial y_{j}} \log |x-y|,
$$

$\Delta_{y} \log |x-y|=2 /|x-y|^{2}$.

Lemma 5. Let $\zeta_{1}, \ldots, \zeta_{n}$ be differential forms in $L_{n-2}^{p}(\Sigma)$ such that $d \zeta_{j}=(-1)^{n-1} v_{j} d \sigma$ on $\Sigma$. One has $\psi \in \mathcal{N}_{p}$ if, and only if,

$$
\psi_{j}=\sum_{h=0}^{m} c_{h} \chi_{\Sigma_{h}} \zeta_{j}+\eta_{j}, \quad j=1, \ldots, n,
$$

where $c_{0}, \ldots, c_{m} \in \mathbb{R}$ and $\eta_{1} \ldots, \eta_{n}$ are weakly closed forms belonging to $L_{n-2}^{p}(\Sigma)$.

Proof. It is easy to construct the differential forms $\zeta_{1}, \ldots$, $\zeta_{n}$. For example, one can take the restriction on $\Sigma$ of the following forms: $\zeta_{1}=(-1)^{n-1} x_{2} d x^{3} \cdots d x^{n}, \zeta_{j}=$ $(-1)^{n-j} x_{1} d x^{2} \cdots \hat{j} \cdots d x^{n}(j=2, \ldots, n)$. We remark that $(37)$ holds if, and only if, the weak differentials $d \psi_{j}$ exist and

$$
d \psi_{j}=(-1)^{n-1} \sum_{h=0}^{m} c_{h} \chi_{\Sigma_{h}} v_{j} d \sigma, \quad j=1, \ldots, n,
$$

that is,

$$
\int_{\Sigma} \psi_{j} \wedge d u_{j}=\sum_{h=0}^{m} c_{h} \int_{\Sigma_{h}} u \cdot v d \sigma, \quad \forall u \in\left[C_{0}^{\infty}\left(\mathbb{R}^{n}\right)\right]^{n}
$$

Let us prove that (39) holds if, and only if,

$$
\begin{array}{r}
\int_{\Sigma_{k}} \psi_{j} \wedge d u_{j}=c_{k} \int_{\Sigma_{k}} u \cdot v d \sigma, \\
\forall u \in\left[C_{0}^{\infty}\left(\mathbb{R}^{n}\right)\right]^{n}, \quad k=0, \ldots, m .
\end{array}
$$

It is obvious that (40) implies (39).

Conversely, suppose that (39) is true. Define $U_{k}^{\varepsilon}=\{x \in$ $\left.\mathbb{R}^{n} \mid \operatorname{dist}\left(x, \Sigma_{k}\right)<\varepsilon\right\}$, where $0<\varepsilon<\min _{0 \leq h<k \leq m} \operatorname{dist}\left(\Sigma_{h}, \Sigma_{k}\right)$. Let $v_{k} \in C_{0}^{\infty}\left(U_{k}^{\varepsilon}\right)$ be such that $v_{k}=1$ in $U_{k}^{\varepsilon / 2}$. Since $v_{k} u \in$ $\left[C_{0}^{\infty}\left(\mathbb{R}^{n}\right)\right]^{n}$, we may write

$$
\int_{\Sigma} \psi_{j} \wedge d\left(v_{k} u_{j}\right)=\sum_{h=0}^{m} c_{h} \int_{\Sigma_{h}} v_{k} u \cdot v d \sigma
$$

and (40) follows immediately.

Suppose now that (39) is true. From (40) it follows that

$$
\int_{\Sigma_{k}} \psi_{j}(y) \wedge d_{y}\left[\gamma_{i j}(x, y)\right]=c_{k} \int_{\Sigma_{k}} \gamma_{i j}(x, y) v_{j}(y) d \sigma_{y},
$$$$
\forall x \notin \Sigma_{k} \text {. }
$$

An integration by parts shows that

$$
\int_{\Sigma_{k}} \psi_{j}(y) \wedge d_{y}\left[\gamma_{i j}(x, y)\right]=0, \quad \forall x \notin \bar{\Omega}_{k} .
$$

Taking the exterior angular boundary value (for the definition of internal (external) angular boundary values see, e.g., [20, page 53] or [21, page 293]), we have

$$
\int_{\Sigma_{k}} \psi_{j}(y) \wedge d_{y}\left[\gamma_{i j}(x, y)\right]=0
$$

a.e. on $\Sigma_{k}$. Arguing as in [9, pages 189-190], this implies that

$$
\int_{\Sigma_{k}} \psi_{j}(y) \wedge d_{y}\left[\gamma_{i j}(x, y)\right]=0
$$

also in $\Omega_{k}$. Summing over $k$ we find

$$
\int_{\Sigma} \psi_{j}(y) \wedge d_{y}\left[\gamma_{i j}(x, y)\right]=0
$$

for every $x \in \mathbb{R}^{n} \backslash \Sigma$ and a.e. on $\Sigma$. In particular $\psi$ is the solution of the singular integral system (28).

Conversely, suppose (28) holds. Arguing again as in [9, pages 189-190], from (28) it follows that

$$
\int_{\Sigma} \psi_{j}(y) \wedge d_{y}\left[\gamma_{i j}(x, y)\right]=0, \quad x \notin \Sigma .
$$

Since $\varepsilon_{j}(x, y)=-\partial_{x_{j}} s(x, y)$, system (11) implies that $\Delta_{x}\left[\gamma_{i j}(x, y)\right]=-(1 / \mu)\left(\partial^{2} / \partial x_{i} \partial x_{j}\right) s(x, y)$. Hence,

$$
\frac{\partial^{2}}{\partial x_{i} \partial x_{j}} \int_{\Sigma} \psi_{j}(y) \wedge d_{y}[s(x, y)]=0, \quad x \notin \Sigma .
$$

Therefore, there exist some constants $a_{0}, a_{1}, \ldots, a_{m}$ such that

$$
\partial_{j} \Psi_{j}(x)= \begin{cases}-a_{h} & x \in \Omega_{h}, h=1, \ldots, m, \\ -a_{0} & x \in \Omega, \\ 0 & x \in \mathbb{R}^{n} \backslash \bar{\Omega},\end{cases}
$$

where

$$
\Psi_{j}(x)=\int_{\Sigma} \psi_{j}(y) \wedge d_{y}[s(x, y)]
$$

Then, on account of Lemma 4 , for every $u \in\left[C_{0}^{\infty}\left(\mathbb{R}^{n}\right)\right]^{n}$,

$$
\begin{aligned}
\int_{\Sigma} \psi_{j} \wedge d u_{j}= & \mu \int_{\mathbb{R}^{n}} \Delta u_{j}(x) d x \int_{\Sigma} \psi_{j}(y) \wedge d_{y}\left[\gamma_{i j}(x, y)\right] \\
& +\int_{\mathbb{R}^{n}} \frac{\partial^{2}}{\partial x_{i} \partial x_{j}} u_{i}(x) d x \int_{\Sigma} \psi_{j}(y) \wedge d_{y}[s(x, y)]
\end{aligned}
$$


The first term of the right hand side vanishes because of (47). As far as the second one is concerned, integrating by parts we get

$$
\begin{aligned}
\int_{\mathbb{R}^{n}} & \frac{\partial^{2}}{\partial x_{i} \partial x_{j}} u_{i}(x) \Psi_{j}(x) d x \\
= & \sum_{h=1}^{m} \int_{\Omega_{h}} \frac{\partial^{2}}{\partial x_{i} \partial x_{j}} u_{i}(x) \Psi_{j}(x) d x \\
& +\int_{\Omega} \frac{\partial^{2}}{\partial x_{i} \partial x_{j}} u_{i}(x) \Psi_{j}(x) d x \\
& +\int_{\mathbb{R}^{n} \backslash \bar{\Omega}_{0}} \frac{\partial^{2}}{\partial x_{i} \partial x_{j}} u_{i}(x) \Psi_{j}(x) d x \\
= & -\sum_{h=1}^{m} \int_{\Sigma_{h}} \partial_{i} u_{i} \Psi_{j} v_{j} d \sigma+\sum_{h=1}^{m} \int_{\Omega_{h}} \partial_{i} u_{i} \partial_{j} \Psi_{j} d x \\
& +\int_{\Sigma} \partial_{i} u_{i} \Psi_{j} v_{j} d \sigma-\int_{\Omega} \partial_{i} u_{i} \partial_{j} \Psi_{j} d x \\
& +\sum_{h=1}^{m} \int_{\Omega_{h}} \partial_{i} u_{i} \partial_{j} \Psi_{j} d x-\int_{\Omega} \partial_{i} u_{i} \partial_{j} \Psi_{j} d x . \\
& -\int_{\Sigma_{0}} \partial_{i} u_{i} \Psi_{j} v_{j} d \sigma+\int_{\mathbb{R}^{n} \backslash \bar{\Omega}_{0}} \partial_{i} u_{i} \partial_{j} \Psi_{j} d x
\end{aligned}
$$

Hence, by (49),

$$
\begin{aligned}
\int_{\mathbb{R}^{n}} & \frac{\partial^{2}}{\partial x_{i} \partial x_{j}} u_{i}(x) \Psi_{j}(x) d x \\
= & -\sum_{h=1}^{m} a_{h} \int_{\Omega_{h}} \partial_{i} u_{i} d x+a_{0} \int_{\Omega} \partial_{i} u_{i} d x \\
= & \sum_{h=1}^{m} a_{h} \int_{\Sigma_{h}} u \cdot v d \sigma+a_{0} \int_{\Sigma} u \cdot v d \sigma \\
= & a_{0} \int_{\Sigma_{0}} u \cdot v d \sigma+\sum_{h=1}^{m}\left(a_{0}+a_{h}\right) \int_{\Sigma_{h}} u \cdot v d \sigma .
\end{aligned}
$$

By setting $c_{0}=a_{0}$ and $c_{h}=a_{0}+a_{h}(h=1, \ldots, m)$ we get the claim.

Remark 6. Lemma 5 shows that the dimension of the kernel $\mathcal{N}_{p}$ is infinite. However, if we consider the quotient space $\mathcal{N}_{p} / \Xi_{p}, \Xi_{p}$ being the space of weakly closed differential forms in $L_{n-2}^{p}(\Sigma)$, we have $\operatorname{dim}\left(\mathcal{N}_{p} / \Xi_{p}\right)=m+1$.
We conclude this section by recalling some properties concerning the following eigenspaces:

$$
\begin{aligned}
\mathscr{V}_{ \pm}= & \left\{\varphi_{k} \in L^{p}(\Sigma): \pm \frac{1}{2} \varphi_{k}(x)\right. \\
& \left.\quad+\int_{\Sigma} F_{k i}(x, y) \varphi_{i}(y) d \sigma_{y}=0, k=1, \ldots, n\right\}, \\
\mathscr{W}_{ \pm}= & \left\{\varphi_{k} \in L^{p}(\Sigma): \mp \frac{1}{2} \varphi_{k}(x)\right. \\
& \left.+\int_{\Sigma} F_{i k}(y, x) \varphi_{i}(y) d \sigma_{y}=0, k=1, \ldots, n\right\},
\end{aligned}
$$

where (see, e.g., [22])

$$
\begin{aligned}
F_{k i}(x, y): & =T_{i, y}^{\prime}\left[\gamma^{k}(x, y)\right] \\
& =-\frac{n}{\omega_{n}} \frac{\left(x_{k}-y_{k}\right)\left(x_{i}-y_{i}\right)\left(x_{j}-y_{j}\right)}{|x-y|^{n+2}} v_{j}(y) .
\end{aligned}
$$

For the proofs of the following two results see [7, Lemma 3.3] and [8, Theorem 3.2], respectively.

Proposition 7. The sets $\mathscr{V}_{+}$and $\mathscr{W}_{-}$are linear subspaces of $L^{1}(\Sigma)$ and

$$
\operatorname{dim}\left(\mathscr{V}_{+}\right)=\operatorname{dim}\left(\mathscr{W}_{-}\right)=1+\frac{n(n+1) m}{2}
$$

A basis of $\mathscr{W}_{-}$is expressed by the fields $\left\{\psi_{i h}, v: i=\right.$ $1, \ldots, n(n+1) / 2, h=1, \ldots, m\}$. The simple layer potentials $v_{i h}$ whose densities are $\psi_{i k}$ such that: $\left.v_{i h}\right|_{\Omega_{k}}=\delta_{h k} \rho_{i}$, $i=1, \ldots, n(n+1) / 2, h, k=1, \ldots, m$, where $\rho_{i}$ are rigid displacement in $\mathbb{R}^{n}$, specifically $\rho_{i}(x)=e_{i}, i=1, \ldots, n$, and, for $i=n+1, \ldots, n(n+1) / 2, \rho_{i}(x)=\left(e_{h} \wedge e_{k}\right) x, h=$ $1, \ldots, n-1, k=h+1, \ldots, n, h[n-(h+1) / 2]+k=i$.

In addition, every $\psi \in \mathscr{W}_{-}$has the property that $\left.v\right|_{\Sigma_{0}}=0$, where $v$ is the simple layer potential with density $\psi$.

Proposition 8. The sets $\mathscr{V}_{-}$and $\mathscr{W}_{+}$are linear subspaces of $L^{1}(\Sigma)$ and

$$
\operatorname{dim}\left(\mathscr{V}_{-}\right)=\operatorname{dim}\left(\mathscr{W}_{+}\right)=\frac{n(n+1)}{2}+m
$$

A basis for $\mathscr{W}_{+}$is expressed by the fields $\left\{\psi_{i}, v \chi_{\Sigma_{h}}: i=\right.$ $1, \ldots, n(n+1) / 2, h=1, \ldots, m\}$, where $\psi_{i}, i=1, \ldots, n(n+1) / 2$ are zero on $\Sigma \backslash \Sigma_{0}$, and such that the simple layer potentials with density $\psi_{i}$ are $n(n+1) / 2$ rigid displacement in $\Omega_{0}$ (linearly independent for $n \geq 3$ ).

Finally, every function $\varphi$ which is the restriction to $\Sigma$ of a rigid displacement belongs to $\mathscr{V}_{-}$.

One recalls that if $\varphi \in\left[L^{1}(\Sigma)\right]^{n}$ belongs to one of the eigenspaces $\mathscr{V}_{ \pm}, \mathscr{W}_{ \pm}$, then $\varphi \in\left[C^{\lambda}(\Sigma)\right]^{n}$. This follows from general results about integral equations (see [8, Lemma 31] and $[7$, page 81$])$. 
Remark 9. We can make the statement of Proposition 8 slightly more precise, saying that the simple layer potentials with density $\psi_{i}$ are $n(n+1) / 2$ rigid displacement in $\Omega_{0}$ linear independent for any $n \geq 2$, unless $n=2$ and $\Sigma_{0}$ is exceptional. Indeed, let us show that if $n=2$ and $\Sigma_{0}$ is not exceptional, such rigid displacements are linearly independent. Let $c_{i}$ be such that

$$
\sum_{i=1}^{3} c_{i} \int_{\Sigma_{0}} \psi_{i}(y) \gamma(x, y) d \sigma_{y}=0, \quad \text { in } \Omega_{0} .
$$

We have also

$$
\int_{\Sigma_{0}} \sum_{i=1}^{3} c_{i} \psi_{i}(y) \gamma(x, y) d \sigma_{y}=0, \quad \text { a.e. } x \in \Sigma_{0} .
$$

Let $\varphi=\sum_{i=1}^{3} c_{i} \psi_{i}$. In view of the equivalence between (1) and (3) of Theorem 3, $\varphi$ has to vanish. Therefore $c_{i}=0$ $(i=1,2,3)$ because of the linearly independence of $\psi_{i}$. On the other hand, if $n=2$ and $\Sigma_{0}$ is exceptional, Theorem 3 shows that the potentials with densities $\left\{\psi_{i}\right\}_{i=1,2,3}$ are linearly dependent.

\section{Reduction of a Certain Singular Integral Operator}

For every $\psi \in L_{1}^{p}(\Sigma)$, let $\Theta_{h}$ be the operator defined by

$$
\begin{array}{r}
\Theta_{h}(\psi)(x)=*\left(\int_{\Sigma} d_{x}\left[s_{n-2}(x, y)\right] \wedge \psi(y) \wedge d x^{h}\right), \\
x \in \Omega,
\end{array}
$$

where $*$ and $d$ denote the Hodge star operator and the exterior derivative, respectively, and $s_{h}(x, y)$ is the double $h$ form introduced by Hodge in [23] as follows:

$$
s_{h}(x, y)=\sum_{j_{1}<\cdots<j_{h}} s(x, y) d x^{j_{1}} \cdots d x^{j_{h}} d y^{j_{1}} \cdots d y^{j_{h}} .
$$

Note that the operator $\Theta_{h}$ satisfies the equation

$$
\partial_{h} \int_{\Sigma} u(y) \frac{\partial}{\partial v_{y}} s(x, y) d \sigma_{y}=-\Theta_{h}(d u), \quad x \in \Omega
$$

for each $u \in W^{1, p}(\Sigma)$, since (see [9, page 187])

$$
\begin{aligned}
& * d \int_{\Sigma} u(y) \frac{\partial}{\partial v_{y}} s(x, y) d \sigma_{y} \\
& \quad=d_{x} \int_{\Sigma} d u(y) \wedge s_{n-2}(x, y), \quad x \in \Omega .
\end{aligned}
$$

Moreover we introduce the operators $\mathscr{H}_{j h}$ defined as

$$
\begin{aligned}
\mathscr{H}_{j h}(\psi)(x)= & \Theta_{h}\left(\psi_{j}\right)(x)-\frac{\delta_{l i j_{3} \cdots j_{n}}^{123 \cdots n}}{(n-2) !} \\
& \times \int_{\Sigma} \partial_{x_{h}} H_{l j}(x, y) \wedge \psi_{i}(y) \wedge d y^{j_{3}} \cdots \wedge d y^{j_{n}},
\end{aligned}
$$

for every $\psi \in\left[L_{1}^{p}(\Sigma)\right]^{n}$, where

$$
H_{l j}(x, y)=\frac{1}{\omega_{n}} \frac{\left(y_{l}-x_{l}\right)\left(y_{j}-x_{j}\right)}{|y-x|^{n}} .
$$

In the sequel $d u$ denotes the vector $\left(d u_{1}, \ldots, d u_{n}\right)$ whose elements are 1 -forms, and $\psi=\left(\psi_{1}, \ldots, \psi_{n}\right) \in\left[L_{1}^{p}(\Sigma)\right]^{n}$.

Lemma 10. Let $(w, q)$ be the double layer hydrodynamic potential of (17)-(18) with density $u \in\left[W^{1, p}(\Sigma)\right]^{n}$. Then, for $x \notin \Sigma$,

$$
\begin{gathered}
\partial_{h} w_{j}(x)=\mathscr{H}_{j h}(d u)(x), \\
q(x)=2 \mu \Theta_{h}\left(d u_{h}\right)(x),
\end{gathered}
$$

where $\mathscr{H}_{j h}$ and $\Theta_{h}$ are given by (60) and (64), respectively.

Proof. Note that, even if one could prove (66)-(67) directly, it seems easier to deduce them from the similar results we have already obtained for the elasticity system (see [16, Section 3]). For $k>(n-2) / n$, let $\stackrel{(k)}{w}$ be the double layer elastic potential with density $u$, that is,

$$
\stackrel{(k)}{w}_{j}(x)=\int_{\Sigma} u_{i}(y) \stackrel{(k)}{L_{i, y}}\left[\stackrel{(k)}{\Gamma}^{j}(x, y)\right] d \sigma_{y},
$$

where $\stackrel{(k)}{L}$ and $\stackrel{(k)}{\Gamma}$ are the stress operator and the Kelvin's matrix associated to the Lamé system $-\Delta u-k \nabla \operatorname{div} u=0$, respectively.

Thanks to [16, Lemma 1], we know that

$$
\partial_{h} \stackrel{(k)}{w}_{j}(x)=\stackrel{(k)}{\mathscr{H}}_{j h}(d u)(x)
$$

where

$$
\begin{aligned}
\stackrel{(k)}{\mathscr{H}}_{j h}(\psi)(x)= & \Theta_{h}\left(\psi_{j}\right)(x)-\frac{\delta_{l i j_{3} \cdots j_{n}}^{123 \cdots n}}{(n-2) !} \\
& \times \int_{\Sigma} \partial_{x_{h}} \stackrel{(k)}{H}_{l j}(x, y) \wedge \psi_{i}(y) \wedge d y^{j_{3}} \cdots d y^{j_{n}} \\
\stackrel{(k)}{H}_{l j}(x, y)= & \frac{k}{\omega_{n}(k+1)} \frac{\left(y_{l}-x_{l}\right)\left(y_{j}-x_{j}\right)}{|y-x|^{n}} \\
& -\frac{1}{k+1} \delta_{l j} s(x, y)
\end{aligned}
$$

and $\Theta_{h}$ is given by $(60)$.

From [16, formula (5)] (where we set $\xi=1$ ), letting $k \rightarrow$ $+\infty$, we get

$$
\begin{aligned}
& \partial_{x_{h}}\left\{L_{i, y}^{(k)}\left[\begin{array}{l}
(k) \\
\Gamma^{j}
\end{array}(x, y)\right]\right\} \\
& \longrightarrow-\frac{n}{\omega_{n}} \partial_{x_{h}}\left\{\frac{\left(y_{i}-x_{i}\right)\left(y_{j}-x_{j}\right)\left(y_{k}-x_{k}\right)}{|x-y|^{n+2}} v_{k}(y)\right\} \\
& =\partial_{x_{h}} T_{i, y}^{\prime}\left[\gamma^{j}(x, y)\right],
\end{aligned}
$$


$x \notin \Sigma$, from which $\left.\partial_{h}{ }^{w} w\right) \partial_{h} w$ as $k \rightarrow+\infty$. Therefore we obtain formula (66) by letting $k \rightarrow+\infty$ in (69). Formula (67) is an immediate consequence of (62) because $\varepsilon_{j}(x, y)=$ $-\partial_{x_{j}} s(x, y)$.

For the next lemma it is convenient to recall here two jump formulas proved in [16, Lemmas 2 and 3].

Let $f \in L^{1}(\Sigma)$. If $\eta \in \Sigma$ is a Lebesgue point for $f$, we get

$$
\begin{aligned}
\lim _{x \rightarrow \eta} \int_{\Sigma} f(y) \partial_{x_{s}} \frac{\left(y_{l}-x_{l}\right)\left(y_{j}-x_{j}\right)}{|x-y|^{n}} d \sigma_{y} \\
=\frac{\omega_{n}}{2}\left(\delta_{l j}-2 v_{j}(\eta) v_{l}(\eta)\right) v_{s}(\eta) f(\eta) \\
\quad+\int_{\Sigma} f(y) \partial_{x_{s}} \frac{\left(y_{l}-\eta_{l}\right)\left(y_{j}-\eta_{j}\right)}{|x-y|^{n}} d \sigma_{y},
\end{aligned}
$$

where the limit has to be understood as an internal angular boundary value, and the integral in the right hand side is a singular integral.

Further, let $\psi \in L_{1}^{p}(\Sigma)$ and write $\psi$ as $\psi=\psi_{h} d x^{h}$ with

$$
v_{h} \psi_{h}=0 \text {. }
$$

Assumption (73) is not restrictive, because, given the 1-form $\psi$ on $\Sigma$, there exist scalar functions $\psi_{h}$ defined on $\Sigma$ such that $\psi=\psi_{h} d x^{h}$ and (73) holds (see [24, page 41]). Then, for almost every $\eta \in \Sigma$,

$$
\lim _{x \rightarrow \eta} \Theta_{h}(\psi)(x)=-\frac{1}{2} \psi_{h}(\eta)+\Theta_{h}(\psi)(\eta),
$$

where $\Theta_{h}$ is given by (60), and the limit has to be understood again as an internal angular boundary value.

Lemma 11. Let $\psi \in L_{1}^{p}(\Sigma)$. Let one write $\psi$ as $\psi=\psi_{h} d x^{h}$ and suppose that (73) holds. Then, for almost every $\eta \in \Sigma$,

$$
\begin{aligned}
\lim _{x \rightarrow \eta} & \frac{1}{(n-2) !} \delta_{l i j_{3} \cdots j_{n}}^{123 \cdots n} \int_{\Sigma} \partial_{x_{s}} H_{l j}(x, y) \wedge \psi(y) \wedge d y^{j_{3}} \cdots \wedge d y^{j_{n}} \\
= & -\frac{1}{2}\left[v_{j}(\eta) \psi_{i}(\eta)+v_{i}(\eta) \psi_{j}(\eta)\right] v_{s}(\eta) \\
& +\frac{1}{(n-2) !} \delta_{l i j_{3} \cdots j_{n}}^{123 \cdots n} \int_{\Sigma} \partial_{x_{s}} H_{l j}(\eta, y) \wedge \psi(y) \wedge d y^{j_{3}} \cdots \wedge d y^{j_{n}},
\end{aligned}
$$

where $H_{l j}$ is defined by (65), and the limit has to be understood as an internal angular boundary value.

Proof. We have

$$
\begin{aligned}
& \frac{1}{(n-2) !} \delta_{l i j_{3} \cdots j_{n}}^{123 \cdots n} \int_{\Sigma} \partial_{x_{s}} H_{l j}(x, y) \wedge \psi(y) \wedge d y^{j_{3}} \cdots \wedge d y^{j_{n}} \\
& \quad=\frac{1}{(n-2) !} \delta_{l i j_{3} \cdots j_{n}}^{123 n} \delta_{r h j_{3} \cdots j_{n}}^{123 \cdots n} \int_{\Sigma} \partial_{x_{s}} H_{l j}(x, y) \psi_{h}(y) v_{r}(y) d \sigma_{y} \\
& \quad=\delta_{r h}^{l i} \int_{\Sigma} \partial_{x_{s}} H_{l j}(x, y) \psi_{h}(y) v_{r}(y) d \sigma_{y} .
\end{aligned}
$$

Hence, by (65) and (72),

$$
\begin{aligned}
\lim _{x \rightarrow \eta} & \frac{1}{(n-2) !} \delta_{l i j_{3} \cdots j_{n}}^{122 \cdots n} \int_{\Sigma} \partial_{x_{s}} H_{l j}(x, y) \wedge \psi(y) \wedge d y^{j_{3}} \cdots \wedge d y^{j_{n}} \\
= & \frac{\delta_{r h}^{l i}}{2}\left(\delta_{l j}-2 v_{j}(\eta) v_{l}(\eta)\right) v_{s}(\eta) v_{r}(\eta) \psi_{h}(\eta) \\
& +\frac{1}{(n-2) !} \delta_{l i j_{3} \cdots j_{n}}^{123 \cdots n} \int_{\Sigma} \partial_{x_{s}} H_{l j}(\eta, y) \wedge \psi(y) \wedge d y^{j_{3}} \cdots \wedge d y^{j_{n}} .
\end{aligned}
$$

Keeping in mind (73), we find

$$
\begin{aligned}
\frac{\delta_{r h}^{l i}}{2}\left(\delta_{l j}-2 v_{j} v_{l}\right) v_{s} v_{r} \psi_{h} & =\left(\frac{1}{2} \delta_{l j} v_{s}-v_{j} v_{l} v_{s}\right)\left(v_{l} \psi_{i}-v_{i} \psi_{l}\right) \\
& =-\frac{1}{2} v_{s} v_{j} \psi_{i}-\frac{1}{2} v_{s} v_{i} \psi_{j},
\end{aligned}
$$

and the result follows.

Lemma 12. Let $\psi=\left(\psi_{1}, \ldots, \psi_{n}\right) \in\left[L_{1}^{p}(\Sigma)\right]^{n}$. Then, for almost every $\eta \in \Sigma$,

$$
\begin{aligned}
& \lim _{x \rightarrow \eta} \mu {\left[2 \delta_{i j} \Theta_{h}\left(\psi_{h}\right)(x)+\mathscr{H}_{i j}(\psi)(x)+\mathscr{H}_{j i}(\psi)(x)\right] v_{i}(x) } \\
&=\mu\left[2 \delta_{i j} \Theta_{h}\left(\psi_{h}\right)(\eta)+\mathscr{H}_{i j}(\psi)(\eta)+\mathscr{H}_{j i}(\psi)(\eta)\right] v_{i}(\eta),
\end{aligned}
$$

$\Theta_{h}$ and $\mathscr{H}$ being as in (60) and (64), respectively, and the limit has to be understood as an internal angular boundary value.

Proof. Let us write $\psi_{i}$ as $\psi_{i}=\psi_{i h} d x^{h}$ with

$$
v_{h} \psi_{\text {ih }}=0, \quad i=1, \ldots, n .
$$

On account of (72) and (74), we infer

$$
\begin{aligned}
\lim _{x \rightarrow \eta} \mu & {\left[2 \delta_{i j} \Theta_{h}\left(\psi_{h}\right)(x)+\mathscr{H}_{i j}(\psi)(x)+\mathscr{H}_{j i}(\psi)(x)\right] v_{i}(x) } \\
= & \mu \Psi_{i j}(\psi)(\eta) v_{i}(\eta) \\
+\mu & {\left[2 \delta_{i j} \Theta_{h}\left(\psi_{h}\right)(\eta)\right.} \\
+ & \left.\mathscr{H}_{i j}(\psi)(\eta)+\mathscr{H}_{j i}(\psi)(\eta)\right] v_{i}(\eta),
\end{aligned}
$$

where

$$
\begin{aligned}
\Psi_{i j}(\psi)= & -\delta_{i j} \psi_{h h}-\frac{1}{2} \psi_{i j}+\frac{1}{2}\left(v_{i} \psi_{s s}+v_{s} \psi_{s i}\right) v_{j} \\
& -\frac{1}{2} \psi_{j i}+\frac{1}{2}\left(v_{j} \psi_{s s}+v_{s} \psi_{s j}\right) v_{i} .
\end{aligned}
$$

By (80) we get $\Psi_{i j}(\psi) v_{i}=-\psi_{h h} v_{j}-\psi_{i j} v_{i} / 2+\psi_{s s} v_{j}+v_{s} \psi_{s j} / 2=$ 0 .

Remark 13. Whenever we consider external boundary values, we have just to change the sign in the first term on the right hand sides in (72), (74), and (75), while (79) remains unchanged. 
Lemma 14. Let $w$ be the double layer potential (17) with density $u \in\left[W^{1, p}(\Sigma)\right]^{n}$. Then $T_{+, j} w=T_{-, j} w=\mu\left[2 \delta_{i j} \Theta_{h}\left(d u_{h}\right)+\right.$ $\left.\mathscr{H}_{i j}(d u)+\mathscr{H}_{j i}(d u)\right] v_{i}$ a.e. on $\Sigma$, where $T_{+} w$ and $T_{-} w$ denote the internal and the external angular boundary limits of $T w$, respectively, and $\Theta_{h}$ is given by (60) and $\mathscr{H}$ by (64).

Proof. It is an immediate consequence of (66), (67), (79), and Remark 13.

Proposition 15. Let $R:\left[L^{p}(\Sigma)\right]^{n} \rightarrow\left[L_{1}^{p}(\Sigma)\right]^{n}$ be the following singular integral operator

$$
R \varphi(x)=-\int_{\Sigma} d_{x}[\gamma(x, y)] \varphi(y) d \sigma_{y}
$$

Let one define $R^{\prime}:\left[L_{1}^{p}(\Sigma)\right]^{n} \rightarrow\left[L^{p}(\Sigma)\right]^{n}$ to be the singular integral operator

$$
\begin{gathered}
R_{j}^{\prime}(\psi)(x)=\mu\left[2 \delta_{i j} \Theta_{h}\left(\psi_{h}\right)(x)+\mathscr{H}_{i j}(\psi)(x)\right. \\
\left.+\mathscr{H}_{j i}(\psi)(x)\right] v_{i}(x) .
\end{gathered}
$$

Then

$$
R^{\prime} R \varphi=\frac{1}{4} \varphi-K^{2} \varphi
$$

where

$$
K \varphi(x)=-\int_{\Sigma} T_{x}[\gamma(x, y)] \varphi(y) d \sigma_{y} .
$$

Proof. Let $v$ be the simple layer potential (15) with density $\varphi \in$ $\left[L^{p}(\Sigma)\right]^{n}$. In view of Lemma 14 , we have a.e. on $\Sigma$

$$
R_{j}^{\prime}(R \varphi)=\mu\left[2 \delta_{i j} \Theta_{h}\left(d v_{h}\right)+\mathscr{H}_{i j}(d v)+\mathscr{H}_{j i}(d v)\right] v_{i}=T_{j} w,
$$

where $w$ is the double layer potential (17) with density $v$. Moreover, if $x \in \Omega$,

$$
\begin{aligned}
w_{k}(x) & =\int_{\Sigma} v_{i}(y) T_{i, y}^{\prime}\left[\gamma^{k}(x, y)\right] d \sigma_{y} \\
& =v_{k}(x)+\int_{\Sigma} \gamma_{i k}(x, y) T_{i}[v(y)] d \sigma_{y},
\end{aligned}
$$

and then, on account of (86),

$$
\begin{aligned}
T w & =\frac{1}{2} T v-K(T v)=\frac{1}{2}\left(\frac{1}{2} \varphi+K \varphi\right)-K\left(\frac{1}{2} \varphi+K \varphi\right) \\
& =\frac{1}{4} \varphi-K^{2} \varphi .
\end{aligned}
$$

\section{The Dirichlet Problem}

Let us consider the Dirichlet problem for the Stokes system

$$
\begin{aligned}
\mu \Delta v=\nabla r & \text { in } \Omega, \\
\operatorname{div} v=0 & \text { in } \Omega, \\
v=f & \text { on } \Sigma,
\end{aligned}
$$

where the given data $f \in\left[W^{1, p}(\Sigma)\right]^{n}$ satisfies the compatibility condition (4).

The aim of the present section is to study the representability of the solution of this problem by means of a simple layer hydrodynamic potential (15)-(16).

By the symbol $\mathcal{S}^{p}$ we mean the class of the simple layer hydrodynamic potentials (15)-(16) with density in $\left[L^{p}(\Sigma)\right]^{n}$. Whenever $n=2$ and $\Sigma_{0}$ is exceptional (see Section 4 ), we say that $(v, r)$ belongs to $\mathcal{S}^{p}$ if, and only if,

$$
\begin{gathered}
v(x)=-\int_{\Sigma} \gamma(x, y) \varphi(y) d \sigma_{y}+c, \quad x \in \Omega, \\
r(x)=-\int_{\Sigma} \varepsilon_{j}(x, y) \varphi_{j}(y) d \sigma_{y}, \quad x \in \Omega,
\end{gathered}
$$

where $\varphi \in\left[L^{p}(\Sigma)\right]^{2}$ and $c \in \mathbb{R}^{2}$.

We will see that condition (4) is not sufficient to prove the existence of the solution in the class $\mathcal{S}^{p}$, but it must be satisfied on each $\Sigma_{j}, j=0,1, \ldots, m$.

We begin by proving the following result.

Theorem 16. Given $\omega \in\left[L_{1}^{p}(\Sigma)\right]^{n}$, there exists a solution $\varphi \in$ $\left[L^{p}(\Sigma)\right]^{n}$ of the singular integral system

$$
-\int_{\Sigma} d_{x}[\gamma(x, y)] \varphi(y) d \sigma_{y}=\omega(x), \quad \text { a.e. } x \in \Sigma,
$$

if, and only if,

$$
\int_{\Sigma} \psi_{i} \wedge \omega_{i}=0
$$

for every $\psi=\left(\psi_{1}, \ldots, \psi_{n}\right) \in\left[L_{n-2}^{q}(\Sigma)\right]^{n}(q=p /(p-1))$ such that the weak differentials $d \psi_{j}$ exist and (38) holds for some real constants $c_{0}, \ldots, c_{m}$.

Proof. Consider the adjoint of $R$ (see (83)), $R^{*}:\left[L_{n-2}^{q}(\Sigma)\right]^{n} \rightarrow$ $\left[L^{q}(\Sigma)\right]^{n}$, that is, the operator whose components are given by

$$
R_{i}^{*} \psi(x)=-\int_{\Sigma} \psi_{i}(y) \wedge d_{y}\left[\gamma_{i j}(x, y)\right] .
$$

Proposition 15 implies that the integral system (92) has a solution $\varphi \in\left[L^{p}(\Sigma)\right]^{n}$ if, and only if,

$$
\int_{\Sigma} \psi_{i} \wedge \omega_{i}=0
$$

for each $\psi=\left(\psi_{1}, \ldots, \psi_{n}\right) \in\left[L_{n-2}^{q}(\Sigma)\right]^{n}$ such that $R^{*} \psi=0$. The result follows from Lemma 5 .

Proposition 17. Given $f \in\left[W^{1, p}(\Sigma)\right]^{n}$, there exists a solution of the BVP

$$
\begin{gathered}
(\widetilde{v}, \widetilde{r}) \in \mathcal{S}^{p}, \\
\mu \Delta \widetilde{v}=\nabla \widetilde{r} \quad \text { in } \Omega, \\
\operatorname{div} \widetilde{v}=0 \quad \text { in } \Omega, \\
d \widetilde{v}=d f \quad \text { on } \Sigma,
\end{gathered}
$$

if, and only if, conditions (3) are satisfied. The density $\varphi$ of the pair $(\widetilde{v}, \widetilde{r})($ see $(15)-(16))$ solves the singular integral system $R \varphi=d f$, where $R$ is given by (83). 
Proof. Clearly, there exists a solution of this BVP if, and only if, there exists a solution $\varphi \in\left[L^{p}(\Sigma)\right]^{n}$ of the singular integral system

$$
-\int_{\Sigma} d_{x}[\gamma(x, y)] \varphi(y) d \sigma_{y}=d f(x), \quad \text { a.e. } x \in \Sigma .
$$

In view of Theorem 16, there exists a solution $\varphi$ of this system if, and only if,

$$
\int_{\Sigma} \psi_{i} \wedge d f_{i}=0
$$

for every $\psi=\left(\psi_{1}, \ldots, \psi_{n}\right) \in\left[L_{n-2}^{q}(\Sigma)\right]^{n}$ satisfying $R^{*} \psi=$ 0 , that is, such that the weak differentials $d \psi_{j}$ exist and (39) holds for some real constants $c_{0}, \ldots, c_{m}$. Equation (39) being true for any $u \in\left[W^{1, p}(\Sigma)\right]^{n}$, we can write

$$
\int_{\Sigma} \psi_{i} \wedge d f_{i}=\sum_{h=0}^{m} c_{h} \int_{\Sigma_{h}} f \cdot v d \sigma
$$

because of a density argument. In view of the arbitrariness of $c_{0}, \ldots, c_{m},(98)$ is satisfied if, and only if, (3) holds.

Proposition 18. Let $a_{h} \in \mathbb{R}^{n}(h=0, \ldots, m)$. Let $\psi_{i k}$, $i=$ $1, \ldots, n, k=1, \ldots, m$, be the elements of the basis of $\mathscr{W}_{-}$given by Proposition 7. The pair

$$
\begin{aligned}
& v_{0}(x)=\sum_{k=1}^{m} \sum_{i=1}^{n}\left(a_{k}^{i}-a_{0}^{i}\right) \int_{\Sigma} \gamma(x, y) \psi_{i k}(y) d \sigma_{y}+a_{0}, \quad x \in \Omega, \\
& r_{0}(x)=\sum_{k=1}^{m} \sum_{i=1}^{n}\left(a_{k}^{i}-a_{0}^{i}\right) \int_{\Sigma} \varepsilon(x, y) \psi_{i k}(y) d \sigma_{y}, \quad x \in \Omega,
\end{aligned}
$$

is the solution of the BVP

$$
\begin{gathered}
\left(v_{0}, r_{0}\right) \in \mathcal{S}^{p}, \\
\mu \Delta v_{0}=\nabla r_{0} \quad \text { in } \Omega, \\
\operatorname{div} v_{0}=0 \quad \text { in } \Omega, \\
v_{0}=a_{h} \quad \text { on } \Sigma_{h}, h=0, \ldots, m .
\end{gathered}
$$

Proof. The pair $\left(v_{0}, r_{0}\right)$ belongs to $\mathcal{S}^{p}$ (for $n=2$, see Remark 9). Obviously it satisfies the Stokes system, and it satisfies the boundary conditions since, thanks to Proposition 7,

$$
\begin{aligned}
\left.v_{0}\right|_{\Sigma_{0}} & =\left.\sum_{k=1}^{m} \sum_{i=1}^{n}\left(a_{k}^{i}-a_{0}^{i}\right) v_{i k}\right|_{\Sigma_{0}}+a_{0}=a_{0}, \\
\left.v_{0}\right|_{\Sigma_{h}} & =\left.\sum_{k=1}^{m} \sum_{i=1}^{n}\left(a_{k}^{i}-a_{0}^{i}\right) v_{i k}\right|_{\Sigma_{h}}+a_{0} \\
& =\sum_{k=1}^{m} \sum_{i=1}^{n}\left(a_{k}^{i}-a_{0}^{i}\right) \delta_{h k} e_{i}+a_{0}=a_{h},
\end{aligned}
$$

for any $h=1, \ldots, m$.
Theorem 19. Given $f \in\left[W^{1, p}(\Sigma)\right]^{n}$, the Dirichlet problem

$$
\begin{gathered}
(v, r) \in \mathcal{S}^{p}, \\
\mu \Delta v=\nabla r \quad \text { in } \Omega, \\
\operatorname{div} v=0 \quad \text { in } \Omega, \\
v=f \quad \text { on } \Sigma,
\end{gathered}
$$

is solvable if, and only if, conditions (3) are satisfied. Moreover the solution $(v, r)$ is unique ( $r$ is unique up to an additive constant).

Proof. Suppose conditions (3) are satisfied. Let $(\widetilde{v}, \widetilde{r})$ be a solution of the problem (96). Since $d \widetilde{v}=d f$ on $\Sigma, \widetilde{v}=f+a_{h}$ on $\Sigma_{h}(h=0, \ldots, m)$ for some $a_{h} \in \mathbb{R}^{n}$. The pair $(v, r)=$ $(\widetilde{v}, \widetilde{r})-\left(v_{0}, r_{0}\right)$, where $v_{0}$ and $r_{0}$ are given by (100), solves the problem (103).

Conversely, if there exists a solution $(v, r)$ of $(103)$, the compatibility condition (4) has to be satisfied. Moreover, for any $j=1, \ldots, m,(v, r)$ is the solution of the Stokes system also in $\Omega_{j}$. Therefore conditions (3) are satisfied for $j=$ $1, \ldots, m$. These, together with (4), imply (3) also for $j=0$. The uniqueness is known [7, Theorem 5.5].

Remark 20. The density $(\varphi, \varepsilon)$ of $(v, r)$ can be written as $(\varphi, \varepsilon)=\left(\varphi_{0}+\lambda_{0}, \varepsilon\right)$, where $\varphi_{0}$ solves the singular integral system (97), and $\left(\lambda_{0}, \varepsilon\right)$ is the density of a simple layer potential which is constant on every connected component of $\sum$.

Remark 21. If $n \geq 3$ or $n=2$ and $\Sigma_{0}$ is not exceptional, denoting by $\varphi$ the density of the simple layer potential (15)(16) obtained in Theorem 19, we have $\varphi$ that solves the integral system of the first kind

$$
-\int_{\Sigma} \gamma(x, y) \varphi(y) d \sigma_{y}=f(x)
$$

on $\Sigma$. Therefore, Theorem 19 can be seen as an existence theorem for the integral system of the first kind (104) in $L^{p}(\Sigma)$.

If $n=2$ and $\Sigma_{0}$ is exceptional, we have the existence of a solution $(\varphi, c) \in\left[L^{p}(\Sigma)\right]^{2} \times \mathbb{R}^{2}$ of the integral equation

$$
-\int_{\Sigma} \gamma(x, y) \varphi(y) d \sigma_{y}=f(x)+c \text { on } \Sigma .
$$

Remark 22. Observe that the solvability of the Dirichlet problem (90) by means of a simple layer potential hinges on the singular integral system (97). Thanks to Proposition 15, the operator $R^{\prime}$ provides a left reduction for such a system. This reduction is not an equivalent one, but, as in [25, pages 253-254], one can show that $R^{\prime}$ is a weakly equivalent reduction (see definition in Section 3). Since the system $R \varphi=$ $d f$ is solvable, we have $R \varphi=d f$ if, and only if, $\varphi$ is solution of the Fredholm system $R^{\prime} R \varphi=R^{\prime} d f$. In this sense, such Fredholm system is equivalent to the problem (103).

In order to obtain a similar integral representation for the solution of the Dirichlet problem (90) when $f$ satisfies the only condition (4), we need to modify the representation of the solution by adding an extra term. 
By $\widetilde{\mathcal{S}}^{p}$ we denote the space of all pairs $(v, r)$ written as

$$
\begin{aligned}
v_{i}(x)= & -\int_{\Sigma} \gamma_{i j}(x, y) \varphi_{j}(y) d \sigma_{y} \\
& +\int_{\Sigma} T_{j, y}^{\prime}\left[\gamma^{i}(x, y)\right] \psi_{j}(y) d \sigma_{y}, \quad i=1, \ldots, n, x \in \Omega, \\
r(x)= & -\int_{\Sigma} \varepsilon_{j}(x, y) \varphi_{j}(y) d \sigma_{y} \\
& +2 \mu \int_{\Sigma} \frac{\partial}{\partial \nu_{y}}\left[\varepsilon_{j}(x, y)\right] \psi_{j}(y) d \sigma_{y}, \quad x \in \Omega,
\end{aligned}
$$

where $\varphi$ and $\psi$ belong to $\left[L^{p}(\Sigma)\right]^{n}$.

Theorem 23. Given $f \in\left[W^{1, p}(\Sigma)\right]^{n}$ satisfying (4), the Dirichlet problem

$$
\begin{gathered}
(v, r) \in \widetilde{\mathcal{S}}^{p}, \\
\mu \Delta v=\nabla r \quad \text { in } \Omega, \\
\operatorname{div} v=0 \quad \text { in } \Omega, \\
v=f \quad \text { on } \Sigma,
\end{gathered}
$$

has one, and only one, solution $(v, r)$ given by

$$
\begin{aligned}
v_{i}(x)= & -\int_{\Sigma} \gamma_{i j}(x, y) \varphi_{j}(y) d \sigma_{y} \\
& +\int_{\Sigma} T_{j, y}^{\prime}\left[\gamma^{i}(x, y)\right] f_{j}(y) d \sigma_{y}, \quad x \in \Omega, \\
r(x)= & -\int_{\Sigma} \varepsilon_{j}(x, y) \varphi_{j}(y) d \sigma_{y} \\
& +2 \mu \int_{\Sigma} \frac{\partial}{\partial v_{y}}\left[\varepsilon_{j}(x, y)\right] f_{j}(y) d \sigma_{y}, \quad x \in \Omega,
\end{aligned}
$$

where $\varphi \in\left[L^{p}(\Sigma)\right]^{n}$ is solution of the integral system of the first kind

$$
\begin{aligned}
& -\int_{\Sigma} \gamma_{i j}(x, y) \varphi_{j}(y) d \sigma_{y} \\
& \quad=\frac{1}{2} f_{i}(x)-\int_{\Sigma} T_{j, y}^{\prime}\left[\gamma^{i}(x, y)\right] f_{j}(y) d \sigma_{y}, \quad \text { a.e. on } \Sigma .
\end{aligned}
$$

Proof. Let $v$ be given by (108); imposing the boundary condition, we get (the symbol $w_{+}\left(w_{-}\right)$stands for the interior (exterior) value of the double layer potential (17) on $\Sigma$ )

$$
-\int_{\Sigma} \gamma_{i j}(x, y) \varphi_{j}(y) d \sigma_{y}=f_{i}(x)-w_{+, i}(x), \quad \text { a.e. } x \in \Sigma \text {. }
$$

In view of Remark 21 such a system is solvable if, and only if,

$$
\int_{\Sigma_{h}}\left(f_{i}-w_{+, i}\right) v_{i} d \sigma=0, \quad h=0, \ldots, m .
$$

On the other hand, because of the jump formulas, we have

$$
\begin{aligned}
f_{i}(x)-w_{+, i}(x) & \\
= & f_{i}(x)-\left(\frac{1}{2} f_{i}(x)+\int_{\Sigma} T_{j, y}^{\prime}\left[\gamma^{i}(x, y)\right] f_{j}(y) d \sigma_{y}\right) \\
& :=\frac{1}{2} f_{i}(x)-w_{i}(x)=-w_{-, i}(x), \quad \text { a.e. } x \in \Sigma .
\end{aligned}
$$

Therefore, conditions (112) become $-\int_{\Sigma_{h}} w_{-} \cdot v d \sigma=0, h=$ $0, \ldots, m$. Since $w_{-}$can be considered as the datum of the interior Dirichlet problem in $\Omega_{h}$, for $h=1, \ldots, m$, we have

$$
\int_{\Sigma_{h}} w_{-} \cdot v d \sigma=0, \quad h=1, \ldots, m
$$

As far as $h=0$ is concerned, first we remark that (4) implies $\int_{\Sigma} w \cdot v d \sigma=0$, because

$$
0=\int_{\Sigma} w_{+} \cdot v d \sigma=\int_{\Sigma}\left(\frac{1}{2} f+w\right) \cdot v d \sigma=\int_{\Sigma} w \cdot v d \sigma
$$

Keeping in mind (4) and (114), this leads to

$$
\begin{aligned}
\int_{\Sigma_{0}} & \left(\frac{1}{2} f-w\right) \cdot v d \sigma \\
= & \frac{1}{2} \int_{\Sigma_{0}} f \cdot v d \sigma-\int_{\Sigma_{0}} w \cdot v d \sigma \\
= & -\frac{1}{2} \sum_{j=1}^{m} \int_{\Sigma_{j}} f \cdot v d \sigma+\sum_{j=1}^{m} \int_{\Sigma_{j}} w \cdot v d \sigma \\
= & \sum_{j=1}^{m} \int_{\Sigma_{j}} w_{-} \cdot v d \sigma=0 .
\end{aligned}
$$

Finally, assume that $(v, r)$ is the solution of (107) with the data $f=0$. The integral representation (108) shows that $(v, r) \in$ $\mathcal{S}^{p}$, and then the uniqueness follows from Theorem 19.

\section{References}

[1] L. Cattabriga, "Su un problema al contorno relativo al sistema di equazioni di Stokes," Rendiconti del Seminario Matematico della Università di Padova, vol. 31, pp. 308-340, 1961.

[2] V. A. Solonnikov, "On estimates of Green's tensors for certain boundary problems," Doklady Akademii Nauk, vol. 130, pp. 988991, 1960 (Russian), Translates in Soviet Mathematics Doklady, vol. 1, pp. 128-131, 1960.

[3] M. Kohr, "A mixed boundary value problem for the unsteady Stokes system in a bounded domain in $\mathbb{R}_{n}$," Engineering Analysis with Boundary Elements, vol. 29, no. 10, pp. 936-943, 2005.

[4] M. Kohr, "The Dirichlet problems for the Stokes resolvent equations in bounded and exterior domains in $\mathbb{R}^{n}$," Mathematische Nachrichten, vol. 280, no. 5-6, pp. 534-559, 2007.

[5] M. Kohr, "The interior Neumann problem for the Stokes resolvent system in a bounded domain in $\mathbb{R}^{n}$," Archives of Mechanics, vol. 59, no. 3, pp. 283-304, 2007. 
[6] M. Kohr, "Boundary value problems for a compressible Stokes system in bounded domains in $\mathbb{R}^{n}$," Journal of Computational and Applied Mathematics, vol. 201, no. 1, pp. 128-145, 2007.

[7] P. Maremonti, R. Russo, and G. Starita, "On the Stokes equations: the boundary value problem," in Advances in Fluid Dynamics, pp. 69-140, Quaderni di Matematica Aracne, Rome, Italy, 1999.

[8] G. Starita and A. Tartaglione, "On the traction problem for the Stokes system," Mathematical Models \& Methods in Applied Sciences, vol. 12, no. 6, pp. 813-834, 2002.

[9] A. Cialdea, "On the oblique derivation problem for the Laplace equation, and related topics," Rendiconti della Accademia Nazionale delle Scienze detta dei XL, vol. 12, no. 1, pp. 181-200, 1988.

[10] G. Fichera, "Una introduzione alla teoria delle equazioni integrali singolari," Rendiconti di Matematica, vol. 17, pp. 82-191, 1958.

[11] S. G. Mikhlin and S. Prössdorf, Singular Integral Operators, Springer, Berlin, Germany, 1986.

[12] A. Cialdea and G. C. Hsiao, "Regularization for some boundary integral equations of the first kind in mechanics," Rendiconti della Accademia Nazionale delle Scienze detta dei XL, vol. 19, pp. 25-42, 1995.

[13] A. Cialdea, V. Leonessa, and A. Malaspina, "On the Dirichlet and the Neumann problems for Laplace equation in multiply connected domains," Complex Variables and Elliptic Equations, vol. 57, no. 10, pp. 1035-1054, 2012.

[14] A. Malaspina, "Regularization for integral equations of the first kind in the theory of thermoelastic pseudo-oscillations," Applied Mathematics, Informatics and Mechanics, vol. 9, no. 2, pp. 29-51, 2004.

[15] A. Malaspina, "On the traction problem in mechanics," Archives of Mechanics, vol. 57, no. 6, pp. 479-491, 2005.

[16] A. Cialdea, V. Leonessa, and A. Malaspina, "Integral representations for solutions of some BVPs for the Lamé system in multiply connected domains," Boundary Value Problems, vol. 2011, aticle 53, 2011.

[17] A. Malaspina, "Regularization of some integral equations of the first kind," AIP Conference Proceedings, vol. 1281, pp. 916-919, 2010.

[18] A. Malaspina, "Integral representation for the solution of Dirichlet problem for the stokes system," AIP Conference Proceedings, vol. 1389, pp. 473-476, 2011.

[19] A. Cialdea, E. Dolce, A. Malaspina, and V. Nanni, "On an integral equation of the first kind arising in the theory of Cosserat," submitted.

[20] A. Cialdea, "A general theory of hypersurface potentials," Annali di Matematica Pura ed Applicata, vol. 168, pp. 37-61, 1995.

[21] V. D. Kupradze, T. G. Gegelia, M. O. Basheleĭshvili, and T. V. Burchuladze, Three-Dimensional Problems of the Mathematical Theory of Elasticity and Thermoelasticity, vol. 25 of NorthHolland Series in Applied Mathematics and Mechanics, NorthHolland Publishing, Amsterdam, The Netherlands, 1979.

[22] O. A. Ladyzhenskaya, The Mathematical Theory of Viscous Incompressible Flow, Gordon and Breach Science Publishers, New York, NY, USA, 1969.

[23] W. V. D. Hodge, "A dirichlet problem for harmonic functionals, with applications to analytic varities," Proceedings of the London Mathematical Society, vol. S2-36, no. 1, pp. 257-303, 1934.

[24] A. Cialdea, "On the finiteness of the energy integral in elastostatics with non-absolutely continuous data," Atti della
Accademia Nazionale dei Lincei. Classe di Scienze Fisiche, Matematiche e Naturali. Rendiconti Lincei IX, vol. 4, no. 1, pp. 35-42, 1993.

[25] A. Cialdea, "The multiple layer potential for the biharmonic equation in $n$ variables," Atti della Accademia Nazionale dei Lincei. Classe di Scienze Fisiche, Matematiche e Naturali. Rendiconti Lincei IX, vol. 3, no. 4, pp. 241-259, 1992. 


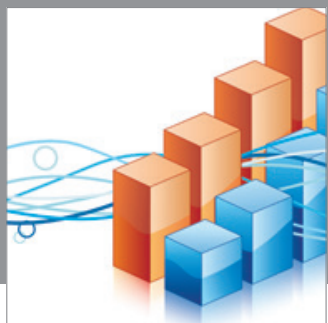

Advances in

Operations Research

mansans

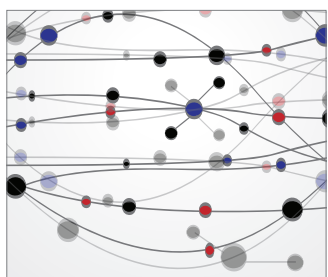

The Scientific World Journal
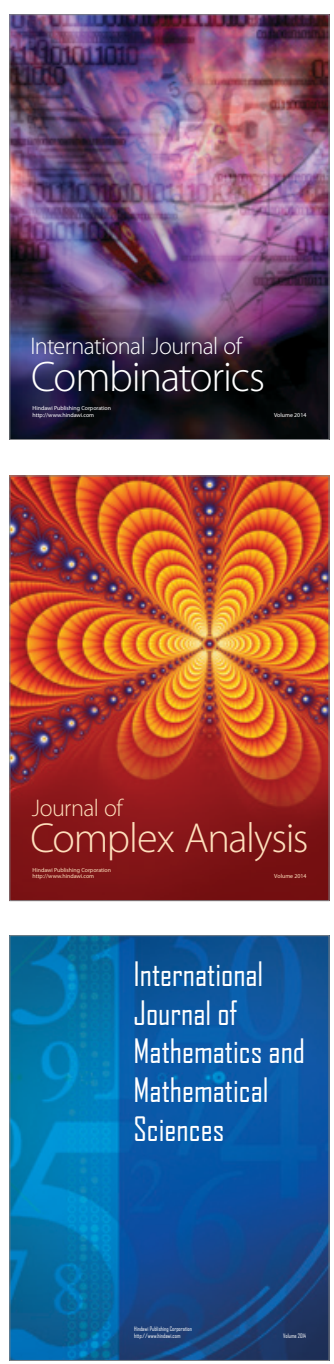
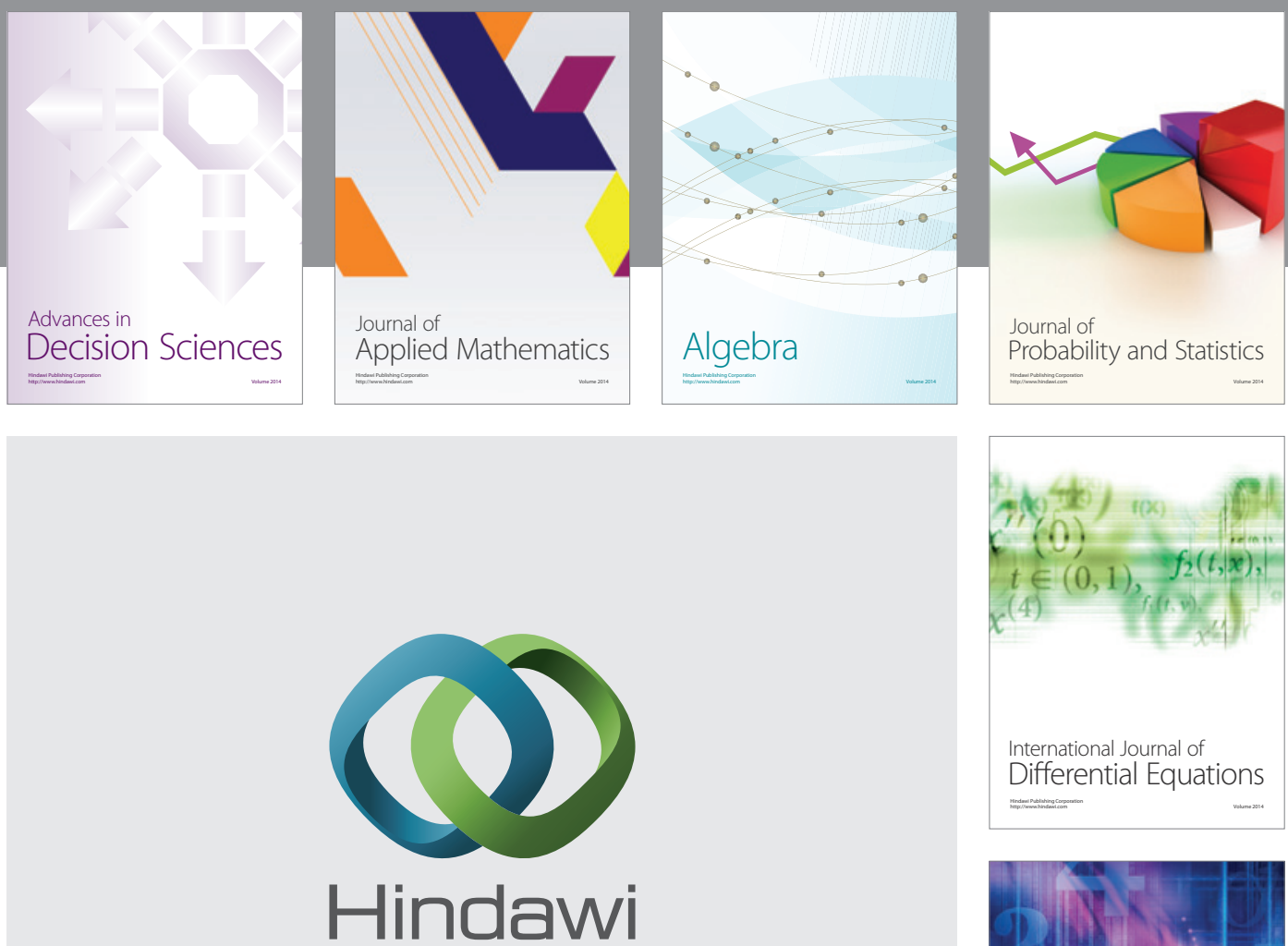

Submit your manuscripts at http://www.hindawi.com
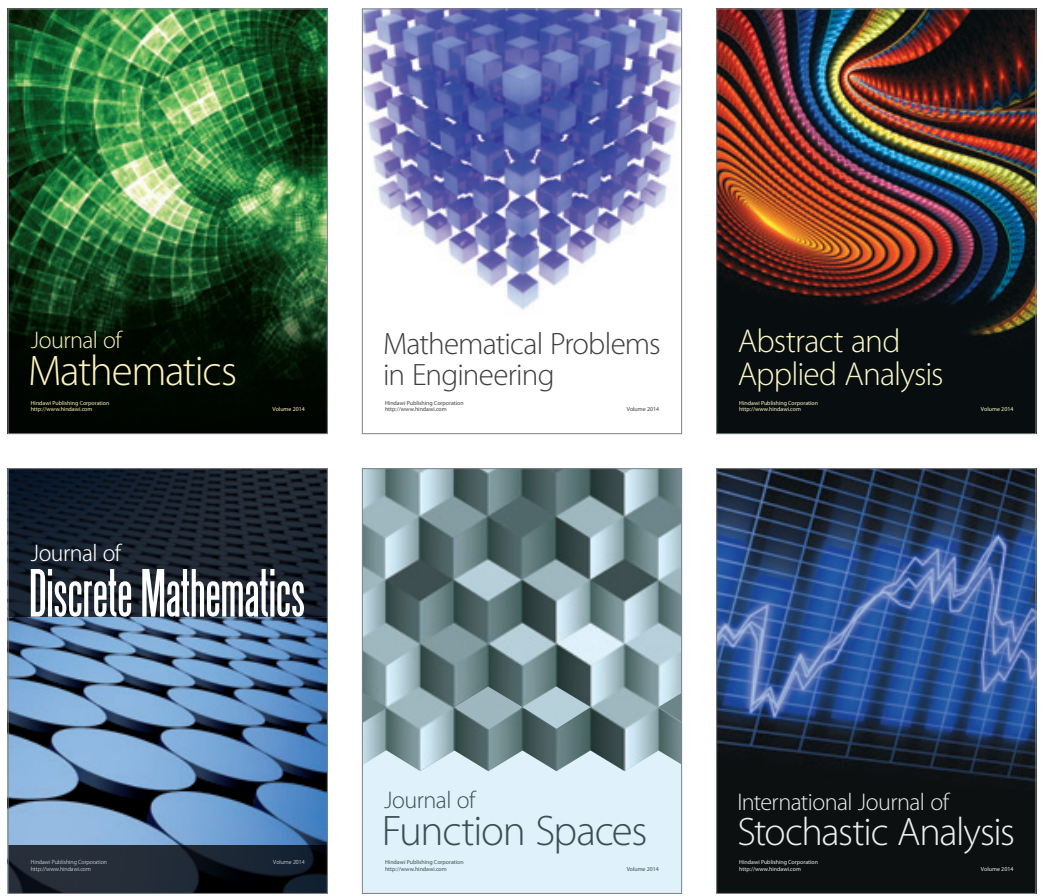

Journal of

Function Spaces

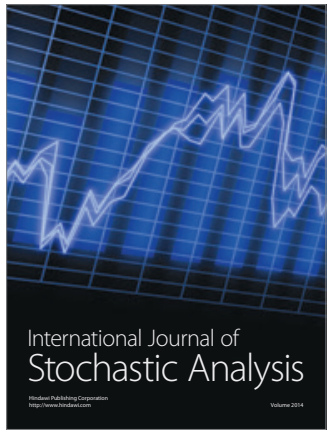

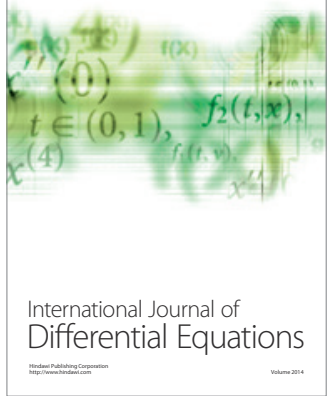
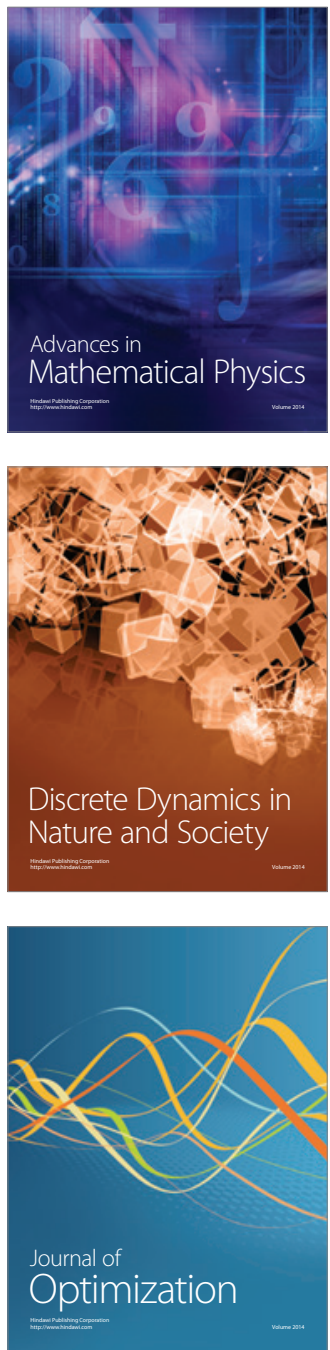